\title{
GRAINE Project: a balloon-borne emulsion gamma-ray telescope
}

\author{
Hiroki Rokujo* \\ Nagoya University \\ E-mail: rokujodflab.phys.nagova-u.ac.jp
}

\section{GRAINE collaboration}

Aichi University of Education, ISAS/JAXA, Utsunomiya University, Okayama University of

Science, Kobe University, and Nagoya University

\begin{abstract}
Observations of cosmic gamma rays emitted from black holes, pulsars, super nova remnants, etc. are important to understand such high-energy objects or phenomena in the universe. Some gamma-ray telescopes loaded on satellites have surveyed the sub-GeV/GeV gamma-ray sky, and contributed to the development of gamma-ray astronomy. On the other hand, new issues have come to light. Especially, observations at the low galactic latitude remain difficult because of a lack of angular resolution. The Gamma-Ray Astro-Imager with Nuclear Emulsion (GRAINE) project aims at precise observations of gamma-ray sources by a balloon-borne gamma-ray telescope. The high-angular resolution gamma-ray telescope, called emulsion telescope, consists of nuclear emulsion films. Its angular resolution is $0.9^{\circ}$ at $100 \mathrm{MeV}$ or $0.1^{\circ}$ at $1 \mathrm{GeV}$. Our second balloon experiment, GRAINE 2015, was performed in 2015 to detect gamma-ray sources, and demonstrate the imaging performance of the emulsion telescope in the $100 \mathrm{MeV}$ energy region. Currently the flight data analysis is on going. In this paper, we checked the imaging performance of the emulsion telescope with an external calibration source. In the energy range above $100 \mathrm{MeV}$, we verified that the emulsion telescope in the flight had the expected angular resolution.
\end{abstract}

The 3rd International Symposium on "Quest for the Origin of Particles and the Universe" 5-7 January 2017

Nagoya University, Japan

\footnotetext{
* Speaker.
} 


\section{Introduction}

Observations of cosmic gamma rays emitted from black holes, pulsars, super nova remnants, etc. are important to understand such high-energy objects or phenomena in the universe. AGILE [W] launched in 2007 and the Large Area Telescope on the Fermi Gamma-ray Space Telescope (Fermi-LAT) [[] launched in 2008 have surveyed the sub-GeV/GeV gamma-ray sky. They have achieved good results and contributed to the development of gamma-ray astronomy: detection of more than 3000 gamma-ray sources [3] ; discovery of cosmic-ray proton acceleration in super nova remnants [四, 回].

On the other hand, new issues have come to light. Observations at the low galactic latitude remain difficult because of a lack of angular resolution, an increase of background flux originating from galactic diffuse gamma rays, etc. Unassociated sources are still included in the high rate in gamma-ray sources detected in the galactic plane [3]. Furthermore, an unexpected gamma-ray excess in the galactic center region was reported [目].

The Gamma-Ray Astro-Imager with Nuclear Emulsion (GRAINE) project aims at precise observations of gamma-ray sources by a balloon-borne gamma-ray telescope. The high-angular resolution gamma-ray telescope, called emulsion telescope, consists of nuclear emulsion films. Because nuclear emulsion can determine angles of electrons and positrons at the beginning point of pair creation interaction $\left(\gamma \rightarrow \mathrm{e}^{+}+\mathrm{e}^{-}\right)$, the angular resolution for gamma rays $(10 \mathrm{MeV}-10$ $\mathrm{GeV})$ is about one order of magnitude higher than that of Fermi-LAT $\left(0.9^{\circ}\right.$ at $100 \mathrm{MeV}, 0.1^{\circ}$ at 1 $\mathrm{GeV})$.

Our second balloon experiment, GRAINE 2015, was performed on May 12th, 2015. It aimed at detecting gamma-ray sources, and demonstrating the imaging performance of the emulsion telescope in the $100 \mathrm{MeV}$ energy region. The balloon was launched from the Alice Springs balloonlaunching station, Australia at 6:33 Australian Central Standard Time (ACST), and reached 37.2 $\mathrm{km}$ altitude at 8:50. At 14:15 the Vela pulsar entered the field of view of the emulsion telescope. It observed the target for about six hours, then the balloon released the gondola at 20:22. The total flight duration in this experiment was 14.4 hours, with 11.5 hours of level flight at 36.0-37.4 km altitude and 4.7-3.8 hPa residual atmospheric pressure. More detail information is described in [四].

In this paper, we report data analysis, especially, performance check of gamma-ray imaging in the GRAINE 2015 experiment.

\section{Detector in GRAINE 2015}

Figure $\mathbb{W}$ shows pictures and a cross sectional view of the emulsion gamma-ray telescope employed in GRAINE 2015. We enlarged the aperture area of the second telescope to $3780 \mathrm{~cm}^{2}$, which is 29 times larger than that of the first telescope. The converter consists of a hundred highsensitive emulsion films. The thicknesses of the plastic layer and the both-side emulsion layers in a film are $180 \mu \mathrm{m}$ and $70 \mu \mathrm{m}$, respectively. The total thickness and the radiation length are $32 \mathrm{~mm}$ and $0.53 X_{0}$, respectively. $34 \%$ of vertical-incident gamma rays convert to electron-positron pairs, and these tracks are recorded in emulsion films. The area of a converter film is $37.8 \mathrm{~cm} \times 25 \mathrm{~cm}$. Four units with the same structure were employed. An alignment unit (two or three emulsion films kept vacuum-packed with an aluminum honeycomb panel) was put on the top of each converter 
unit. The alignment unit is the standard surface of the detector system, and each angle of track recorded in the converter is calibrated by high momentum tracks penetrating both the alignment unit and the converter. The multi-stage shifter system as time stamper[8] was put at the bottom of the converter. 2-4 emulsion films were mounted on each movable stage. Three stages are driven by stepping motors. In the whole of the observation, they slide cyclically like an analog clock, and create independent combinations of the stage position. The energy measurement for multi-GeV gamma-ray events was performed by the analysis of the calorimeter. It has the sandwich structure of sixteen emulsion films and fifteen $1 \mathrm{~mm}$-thick stainless steel plates. The thickness and radiation length were $19.3 \mathrm{~mm}$ and $0.90 X_{0}$, respectively. The balloon also carried three star trackers as attitude monitors, a balloon-style pressure vessel to maintain the vacuum-packed emulsion chamber, and several sensors (for GPS, temperature, pressure, etc.) as its payload.
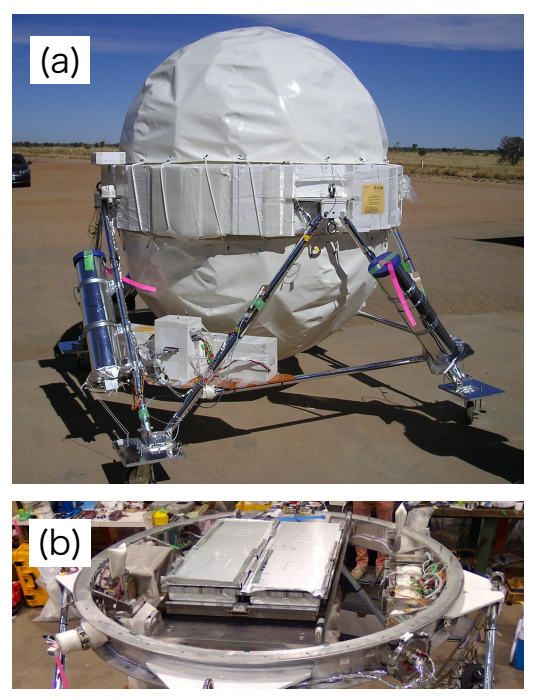

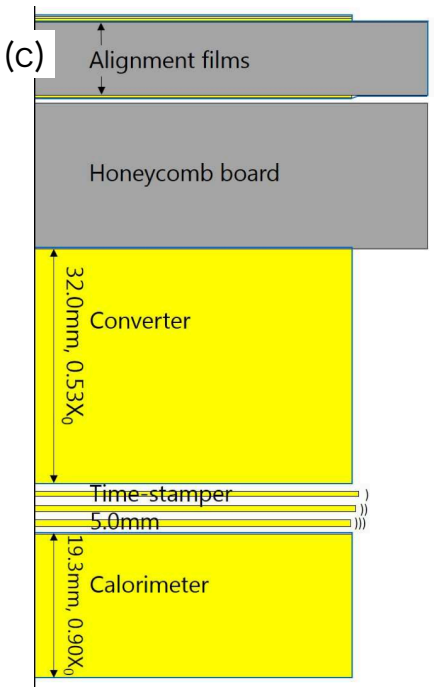

Figure 1: (a) Picture of gondola. A spherical container (the inside diameter is $1.6 \mathrm{~m}$ ) is a balloon-style pressure vessel. Three star cameras as the attitude monitors were mounted on the trusses (one of them was on the other side). (b) Inside view of the pressure vessel. At the center of a ring, the emulsion gamma-ray telescope was put. The total aperture area of four units was $3780 \mathrm{~cm}^{2}$. (c) Cross sectional view of the emulsion gamma-ray telescope. It consists of alignment films, the converter, and time stamper, and the calorimeter.

\section{Imaging performance check}

Data taking of emulsion films was done by the latest scanning system, Hyper Track Selector (HTS), which was developed in Nagoya University [Q]. The first practical data taking of HTS went smoothly and it took about three months to finish $41 \mathrm{~m}^{2}$ of the converter films and the time stamper films.

The analysis of gamma-ray event $\left(\gamma \rightarrow e^{+}+e^{-}\right)$selection is described in [ए]]. The density of the number of tracks read out by HTS was 400 tracks $/ \mathrm{mm}^{2}$ in each film. After event selection, the density decreased $\sim 1 / 2000$. In the current conventional selection, the event-pickup efficiency in the converter was estimated at $65 \%$ and $83 \%$ for $100 \mathrm{MeV}$ and $200 \mathrm{MeV}$ gamma rays, respectively. So far, $75 \%$ of data process (data taking by the scanning system, and gamma-ray event detection in the offline process) were finished.

Figure $\square$ shows pictures of the gondola hanged by a crane truck and an aluminum plate, called a launching plate, that connected the payload to the huge balloon. The crane held the launching 
plate and released it at the timing of launching. After launching, the plate existed $4.4 \mathrm{~m}$ above the emulsion telescope. Cosmic-ray hadrons, mainly protons, were exposed to the plate at the observation altitude. When hadrons interact with the materials, secondary particles including gamma rays (via $\pi^{0} \rightarrow 2 \gamma$ ) are created. Thus, the launching plate became an external gamma-ray source for calibration of the emulsion telescope in the flight.

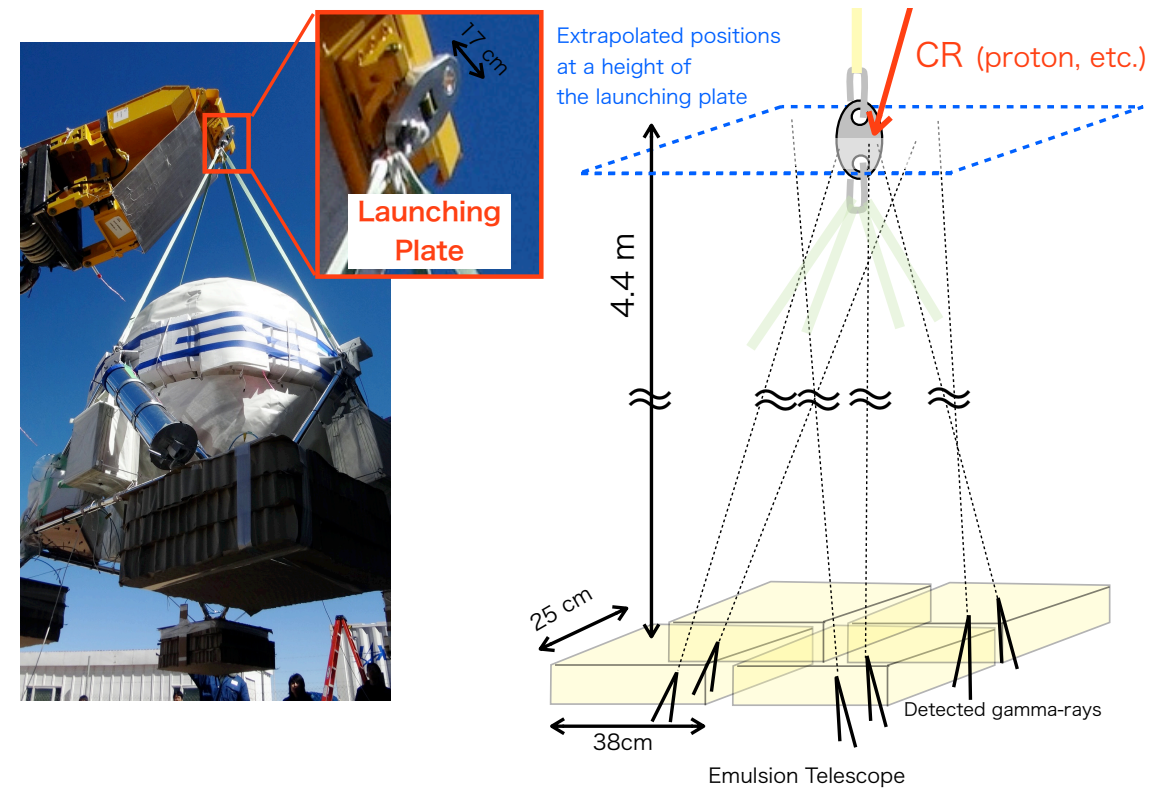

Figure 2: Left pictures show the gondola in GRAINE 2015 and the launching plate. Right schematic view shows the positional relation of the emulsion telescope and the launching plate. A square drawn with blue dotted lines indicates a plane at a height of the launching plate.

The gamma-ray image of the launching plate is not created by the angular distribution of detected gamma-ray events, because the aperture size of the emulsion telescope is not negligible, compared with the distance to the launching plate. So we mapped the extrapolated positions, which are cross points of gamma-ray vectors and a plane at a height of the launching plate. Figure B shows the distributions of extrapolated positions. The energy threshold was set at $100 \mathrm{MeV}$. Left image is a result of counting the number of events with running bins (5-cm radius circle). A clear excess of the number of events was observed and we succeeded in imaging the launching plate by gamma rays. The size of cross section of the plate is $17 \mathrm{~cm}$, and the image extends with the almost same size. We can't discuss the exact point spread function of the emulsion telescope from this image. However, we verified that the emulsion telescope had the angular resolution $\left(\frac{7 \mathrm{~cm}}{440 \mathrm{~cm}} \simeq 0.016 \mathrm{rad}=0.9^{\circ}\right)$ as good as that of the expected performance in $100 \mathrm{MeV}$ region.

\section{Summery and Prospects}

The GRAINE project aims at precise observations of gamma-ray sources with a balloon-borne emulsion gamma-ray telescope. In a test experiment performed in 2015 (the flight duration was 14 hours), the emulsion telescope with $0.38 \mathrm{~m}^{2}$ aperture area was employed to demonstrate its imaging performance. Recovered emulsion films were read out by the latest emulsion scanning system, 

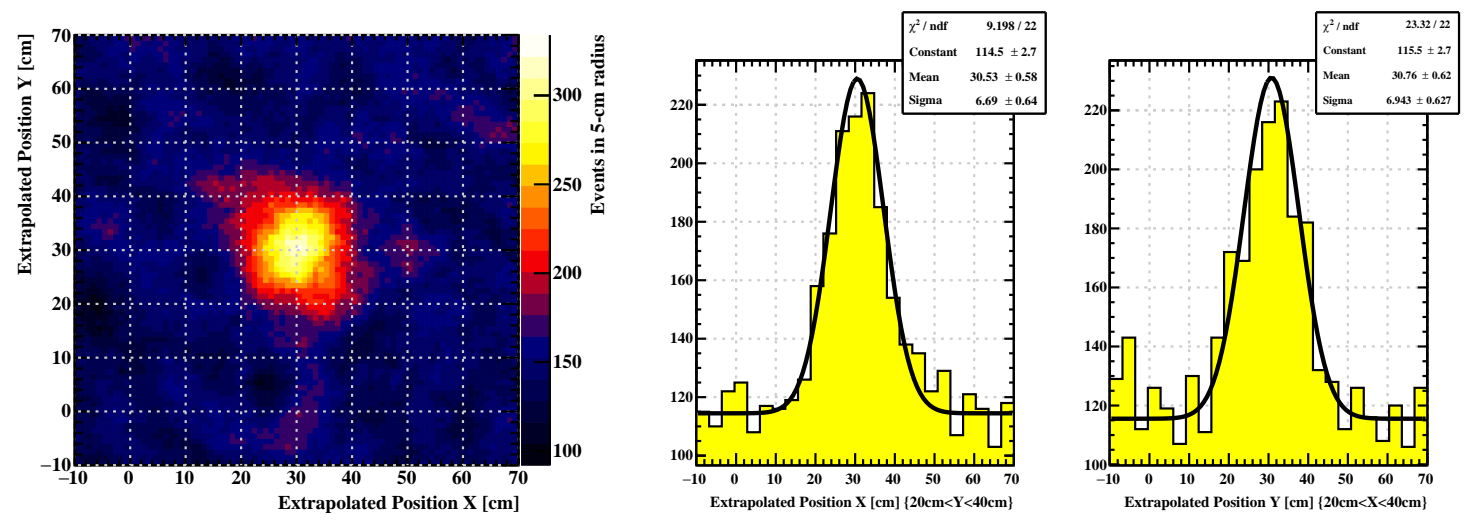

Figure 3: Left image shows an extrapolated position distribution as a result of counting the number of events with running bins (5-cm radius circle). Center and right figures show extrapolated position distributions in $\mathrm{X}$ and $\mathrm{Y}$ projections.

HTS, in Nagoya University and the analysis of gamma-ray event selection almost finished. As we mentioned above, we checked the imaging performance of the emulsion telescope. A gamma-ray image of an external calibration source, the launching plate, was observed, and we succeeded in imaging the launching plate by detected gamma-ray events In the energy range above $100 \mathrm{MeV}$, we verified that the emulsion telescope in the flight had the expected angular resolution. The event rate of gamma rays from the launching plate will be examined in the future.

\section{References}

[1] M. Tavani et al., Astron. Astrophys. 502, 995 (2009).

[2] W. B. Atwood et al., Astrophys. J. 697, 1071 (2009).

[3] F. Acero, et al., The Astrophysical Journal Supplement Series 218(2), 23, (2015).

[4] A. Giuliani, et al., The Astrophysical Journal Letters 742(2), L30, (2011).

[5] M. Ackermann, et al., Science 339(6121), 807-811, (2013).

[6] T. Daylan, et al. Physics of the Dark Universe 12 ,1-23, (2016).

[7] S. Takahashi et al., Prog. Theor. Exp. Phys. 2016, 073 F01 (2016).

[8] S. Takahashi et al., Nucl. Instrum. Meth. A 620, 192 (2010).

[9] M. Yoshiomto et al., to be published in Prog. Theor. Exp. Phys.

[10] H. Rokujo et al., Proceedings of ISVHECRI 2016 to be published in EPJ Web of Conferences. 